\title{
HER2-positive breast cancer - Available anti-HER2 therapies and new agents under investigation
}

\author{
Martyna Zielińska ${ }^{1, A-D \oplus}$, Natalia Zarankiewicz ${ }^{1, B-C}{ }^{\oplus}, K^{2}$ Katarzyna Kosz ${ }^{1, B-C \oplus}$, \\ Aleksandra Kuchnicka ${ }^{1, \mathrm{~B}-\mathrm{C} \oplus}$, Bogumiła Ciseł2,E-F® \\ 'Students' Scientific Association, Oncological Surgery Department, Medical University, Lublin, Poland \\ ${ }^{2}$ Oncological Surgery Department, Independent Public Teaching Hospital No. 1, Medical University, Lublin, Poland \\ A - Research concept and design, B - Collection and/or assembly of data, C - Data analysis and interpretation, \\ $D$ - Writing the article, E - Critical revision of the article, F- Final approval of article
}

\begin{abstract}
Zielińska M, Zarankiewicz N, Kosz K, Kuchnicka A, Ciseł B. HER2-positive breast cancer - Available anti-HER2 therapies and new agents under investigation. J Pre-Clin Clin Res. 2020; 14(2): 44-48. doi: 10.26444/jpccr/122068
\end{abstract}

\begin{abstract}
Introduction and objectives. Breast cancer $(\mathrm{BC})$ is the most common malignancy and the leading cause of cancer death among women. About 15-20\% of all BCs are HER2-positive. Proper assessment of HER2 status is crucial to choose appropriate treatment. The review summarizes data on anti-HER2 drugs used to treat HER2-positive BC and provides basic information on new agents under investigation.

Brief description of the state of knowledge. Specific HER2-targeting drugs are available or are being evaluated in clinical trials. Anti-HER2 agents include: monoclonal antibodies, tyrosine kinase inhibitors, antibody-drug conjugates, bispecific antibodies, PI3K/AKT/mTOR inhibitors and heat shock protein 90 inhibitors, HER2-targeting vaccines and CDK4/6 inhibitors. The advent of anti-HER2 therapies increased the time of progression free survival and overall survival in BC patients.

Results. Final analysis of the CLEOPATRA trial shows that the combination of trastuzumab, pertuzumab and taxane significantly improved outcomes in metastatic HER2-positive BC and it is currently preferred first-line treatment. The recommended second-line treatment is based on trastuzumab emtansine or on the combination of lapatinib and capecitabine. Some promising agents such as margetuximab or trastuzumab deruxtecan are still under investigation.

Conclusions. Anti-HER2 directed treatment undoubtedly improves outcomes among patients with HER2-positive BC. Access to drugs such as trastuzumab, pertuzumab, lapatinib and T-DM1 improves prognosis even in patients with advanced disease. Further studies and clinical trials on novel anti-HER2 therapies are required. Nevertheless, BC treatment is becoming more effective and, hopefully, one day it may be possible to cure patients even with metastases.
\end{abstract}

\section{Key words}

breast cancer, targeted therapies, HER2-positive breast cancer, HER2-targeting treatment, anti-HER2 drugs

\section{INTRODUCTION}

Breast cancer $(\mathrm{BC})$ is currently the most common malignant tumour in women worldwide. The World Health Organization (WHO) report from 2018 states that over 2 million new BC cases are diagnosed each year globally. This fact makes BC the most commonly diagnosed female cancer (24.2\%). Moreover, it is also the leading cause of cancer death among women (15\%) [1].

The human epidermal growth factor receptor 2 (HER2) is a membrane tyrosine kinase. Its activation impinges on cell multiplication, survival, mobility and adherence. It is estimated that HER2 over-expression occurs in around 15-20\% of all BC cases. HER2-positive BCs are deemed to be more aggressive and have poorer outcomes [2].

Proper evaluation of potential HER2 over-expression determines choosing the appropriate treatment for breast cancer patients. False positive results of HER 2 status may lead to unnecessary and ineffective treatment, while false negative results may cause omitting advanced, directed therapy [3]. According to the guidelines from 2013, it is recommended

Address for correspondence: Martyna Zielińska, Students' Scientific Association, Oncological Surgery Department, Medical University, Lublin, Poland, Poland E-mail: martynazielinska52268@gmail.com

Received: 24.03.2020; accepted: 06.05.2020; first published: 22.05.2020 that HER2 testing should be performed in each new case of invasive $\mathrm{BC}$ as well in the case of recurrence if the specimen is accessible. There are several methods of evaluating HER2 amplification, although at present only two are validated for clinical practice [5] (Tab. 1).

Table 1. Methods of assesing HER2 amplification

\begin{tabular}{ll}
\hline Method & Use in clinical practice \\
\hline Immuno-histochemistry (IHC) & Authorized \\
\hline In situ hybridization (ISH) & Authorized \\
\hline PAM50 test & Not validated \\
\hline mRNA expression profiling & Not validated \\
\hline Mass spectrometry & Not validated \\
\hline Serum HER2 levels & Not validated \\
\hline
\end{tabular}

\section{OBJECTIVE}

The aim of the review is to summarize available data on anti-HER2 drugs used in HER2-positive BC treatment and provide basic information on new agents under investigation in several clinical trials. The analysis concerned publications mainly from 2012-2019. The data was collected from PubMed 
and websites such as ClinicalTrials.gov or the World Health Organization website.

\section{DESCRIPTION OF THE STATE OF KNOWLEDGE}

HER2 targeting treatment. The invention of anti-HER2 directed treatment undoubtedly improved the outcomes among patients with HER2-positive BC. Due to applying antiHER2 drugs in treatment, the majority of early BC patients are currently being successfully treated. There is also notable improvement in the time of progression free survival (PFS) and overall survival (OS) among HER2-positive metastatic breast cancer (MBC) patients [6]. Currently available drugs vary in mechanisms of action; however, these drugs can be generally divided into two main categories because of the mode of action. The first category contains agents using the over-expression of HER2 to identify cancer cells, in order that the delivery of anticancer effectors is carried out with great selectivity. The second category involves drugs that inhibit the oncogenic signaling pathways of HER2, resulting in decreasing cancer cell proliferation [3].

Monoclonal antibodies (mAbs). Anti-HER2 monoclonal antibodies use the expression of HER2 as a special target on tumour tissue. Only trastuzumab and pertuzumab remain approved mAbs for HER2-positive BC treatment. The third $\mathrm{mAb}$ being presently under investigation is margetuximab.

Trastuzumab was introduced for the first time for the HER2-positive MBC in 1998. This was a consequence of a randomized trial which indicated that adding trastuzumab to standard chemotherapy resulted in the improvement of PFS and OS in HER2-positive MBC patients [7]. Despite the fact that monotherapy with trastuzumab does not show significant efficacy, it definitely improves the efficacy of chemotherapy. Slamon et al. conducted a study in which patients during the first-line treatment were segregated into two groups: treated with chemotherapy alone versus with the addition of trastuzumab. The results showed a significant improvement in both PFS (7.4 vs. 4.6 months; $\mathrm{P}<0.001)$ and OS ( 25.1 vs. 20.3 months; $\mathrm{P}=0.01)$ in patients treated with the addition of trastuzumab. The most significant side-effect observed during 30 months of median follow-up was cardiac dysfunction, especially in combination with trastuzumab, anthracycline and cyclophosphamide (27\%) compared to anthracycline and cyclophosphamide alone (8\%) and trastuzumab and paclitaxel (8\%) compared to paclitaxel alone (1\%) [8]. In subsequent years, other researchers tested different combinations of trastuzumab with paclitaxel/ docetaxel and carboplatin or vinorelbine. These combinations can be also taken into consideration while deciding on firstline treatment [6]. German Breast Group 26 tested the use of trastuzumab with capecitabine in comparison to capecitabine alone in HER2-positive BC patients after progression during trastuzumab treatment. The findings of this study showed an improvement in overall response and PFS, although not statistically significant in OS [9].

Pertuzumab is humanized $\mathrm{mAb}$ varying from trastuzumab due to binding to a distinct HER2 epitope [6]. It prevents homodimer and heterodimer formations and blocks HER2/ HER3 heterodimers which activate cancer cell proliferation and survival [10]. The study using pertuzumab alone compared to pertuzumab with cytostatics in patients with progression after therapy with trastuzumab showed no desired effect [11]. The CLEOPATRA trial tested the addition of pertuzumab to the basic therapy scheme which consists of docetaxel and trastuzumab. The control group was treated with trastuzumab, docetaxel and placebo and in the research group placebo was replaced by pertuzumab. The addition of pertuzumab to standard treatment resulted in prolongation of both PFS (18.5 vs. 12.4 months, $\mathrm{P}<0.001)$ and OS (56.5 vs. 40.8 months, $\mathrm{P}<0.001)$ in a median follow-up of 50 months $[12,13]$. The final data of the CLEOPATRIA trial, published in 2019 , states that $37 \%$ of patients treated in experimental arm were still alive, whereas only $23 \%$ survived in the control group. Using pertuzumab resulted in 57.1 months of median OS [14]. Pertuzumab improved the efficacy of trastuzumab. At the present time, trastuzumab with a taxane and pertuzumab remain the first-line treatment in patients with HER2-positive MBC.

Margetuximab is a novel antibody derived from trastuzumab and binding to the same epitope. This $\mathrm{mAb}$ has an optimized Fc domain which enables binding to the Fc囚 receptor IIIA (CD16A) forms with high and low chemical attraction. Moreover, it demonstrates reduced affinity to an inhibitory receptor Fc区RIIB (CD32B). These properties of margetuximab enable its binding to effector cells with greater efficacy and enhance antibody-dependent cellular cytotoxicity (ADCC) [10]. Margetuximab alone showed a promising activity and it was generally well tolerated [15]. The SOPHIA trial is currently ongoing. During this research, authors tested the combination of chemotherapy with trastuzumab versus with margetuximab as a third-line treatment in HER2-positive BC [16].

Tyrosine kinase inhibitors (TKIs). Tyrosine kinase inhibitors are small agents which preclude phosphorylation and block the pathways of HER2 signalling. Consequently, this results in a decrease of cancer cell proliferation and promotes apoptosis [17]. Among TKIs, lapatinib and neratinib are registered. Other representatives of this group have been evaluated in several clinical trials.

Lapatinib is the first known TKI used in the treatment of breast cancer, inhibiting both HER2 and EGFR kinases. Geyer et al. investigated lapatinib plus capecitabine in comparison to capecitabine alone in patients suffering from HER2-positive breast cancer who experienced progression on trastuzumab. The results showed PFS enhancement $(8.4$ vs. 4.4 months; $\mathrm{P}<0.001)$ by adding lapatinib to capecitabine therapy [18].

Second-generation TKIs are characterized by a higher chemical attraction to the kinase domain and possibly a wider range of activity.

Neratinib is the first representative, which irreversibly inhibits pan-HER receptor tyrosine kinases [19]. One study reported that neratinib monotherapy showed that in patients non-treated previously with trastuzumab, 16-week PFS was greater compared to women with previous trastuzumabbased therapy (78\% vs. 59\%). The most common side-effect of neratinib was diarrhea (13-30\% of patients) [20]. Results of another clinical trial assessing the influence of neratinib- 
capecitabine treatment after trastuzumab showed 40.3 weeks of median PFS in patients not previously treated with lapatinib, and 35.9 weeks in patients after lapatinib therapy [21]. The NEfERT-T Randomized Clinical Trial reported that as the first-line therapy, trastuzumab-paclitaxel out-performed neratinib-paclitaxel, although it showed that neratinibpaclitaxel may decrease the incidence of central nervous system progression. However, large-scale randomized trials are required to confirm these results [22].

The ExteNET trial investigated neratinib as the next treatment after trastuzumab-based line in patients with HER2-positive early BC. The results showed a reduction of $33 \%$ of invasive recurrence risk in a three years median follow-up [23]. Based on these results, the FDA recently authorized neratinib for patients with HER2-positive early $\mathrm{BC}$ as an additional adjuvant treatment [10]. Another study, the NALA trial, is evaluating the use of lapatinib versus neratinib, both in combination with capecitabine in HER2positive MBC patients [24].

Afatinib is the next second generation TKI. The Lux-Breast III trial evaluated afatinib-containing treatments among patients with HER2-positive metastatic breast cancer and brain metastasis. Benefits of afatinib therapy did not differ from other choices of treatments. Moreover, afatinib caused more adverse effects and was less well tolerated [25]. In several studies, adverse effects of afatinib, such as diarrhea or rush, were the reason to discontinue investigation of this agent, especially as there was insufficient clinical benefit. No clinical trial on the use of afatinib in HER2-positive BCs are being undertaken at present [19].

Tucatinib - an ONT-380 molecule, is another secondgeneration TKI under clinical evaluation. It selectively inhibits HER2, with no substantial influence on HER1. The results of the phase I trial on HER2-positive MBC patients indicate a clinical benefit rate (CBR) of $27 \%$. At present, this drug causes less severe adverse effects than neratinib or afatinib [19]. The combination of trastuzumab and capecitabine, with or without tucatinib, is currently being evaluated in the HER2CLIMB trial, which is addressed to patients suffering from HER2-positive BC with and without brain metastases [26]. Another study tested tucatinib combined with T-DM1 in HER2-positive MBC patients which included 30 patients with brain metastases. The brain-specific overall response rate was $36 \%$, median PFS - 6.7 months. Pyrotinib and poziotinib are two more novel TKIs under investigation in clinical trials [10].

Antibody-drug conjugates (ADCs) consist of three elements: $\mathrm{mAb}$, linker, chemotherapy payload. Specific antibody recognizes targeted cells and a payload attacks these cells in several mechanisms. In $\mathrm{BC}$ treatment, the $\mathrm{mAb}$ used in designing ADCs is usually trastuzumab [3].

Trastuzumab emtansine (T-DM1) is currently the only ADC registered for HER2-positive BC treatment. It consists of trastuzumab and maytansine derivate (DM1), a cytotoxic agent that inhibits mitosis through disrupting microtubules in cancer cells [10]. The EMILIA trial compared the use of T-DM1 and lapatinib-capecitabine in women after progression on trastuzumab-taxane. Analysis showed both PFS and OS were longer with T-DM1 (9.6 months and 29.9 months, respectively) than with lapatinib-capecitabine (6.4 months and 25.9 months, respectively) [27,28]. According to these results, in 2013, the FDA approved T-DM1 for treatment in HER2-positive MBC patients who have progressed on therapy with trastuzumab and docetaxel [3]. This is currently the standard in second-line therapy for patients with HER2positive BC. In compliance with the results of the TH3RESA trial, T-DM1 could be an option for the treatment of patients who experience progression beyond second-line HER2directed treatment if they had not yet received T-DM1 [29]. However, based on the findings of the MARIANNE trial, T-DM1 in not preferred as a first-line treatment. Neither T-DM1 alone nor T-DM1-pertuzumab showed superiority to trastuzumab-taxane [30].

Trastuzumab deruxtecan (DS-8201a), another ADC, is presently under investigation. It comprises anti-HER2 monoclonal antibody trastuzumab and exatecan derivative, which inhibits topoisomerase I. Results of preclinical studies showed that DS-8201a is active not only against HER2-positive tumours, but also against low HER2-expressing cancers [10]. This fact makes DS-8201a superior to T-DM1, which does not show anti-tumour activity if tumours show a low expression of HER2. The overall response rates (ORR) after administering trastuzumab-deruxtecan among prior treated $\mathrm{MBC}$ patients with HER2 over-expression and in low HER2-expressing cases, were $54.5 \%$ and $50 \%$, respectively. It was relatively safe because it mainly caused nausea, decreased appetite and vomiting [31]. Currently, trastuzumab deruxtecan is being investigated in two phase III clinical trials - DESTINYBreast02 and DESTINY-Breast03 [32, 33].

SYD985 is another investigated ADC, based on trastuzumab linked to prodrug DUBA SYD 986 (seco-DUocarmycinhydroxyBenzamide-Azaindole). Its mechanism of action is based on causing irreversible DNA alkylation and consequently death of both dividing and non-dividing cells of a tumour and of neighboring tumour cells. Preclinical results of SYD985 in BC are impressive [3, 10]. The development of this drug is ongoing in phase III TULIP trial [34]. Other ADCs are also currently under investigation in several clinical trials [35-39] (Tab. 2).

Table 2. ADCs under investigation

\begin{tabular}{lcc}
\hline Drug & ClinicalTrials.gov Identifier & Phase \\
\hline Trastuzumab deruxtecan (DS-8201a) & NCT03523585 & Phase 3 \\
\hline SYD985 & NCT03529110 & Phase 3 \\
\hline RC48-ADC & NCT03262935 & Phase 3 \\
\hline PF-06804103 & NCT02881138 & Phase 1 \\
\hline ARX788 & NCT03284723 & Phase 1 \\
\hline MED14276 & NCT02512237 & Phase 1 \\
\hline XMT-1522 & NCT02576548 & Phase 1 \\
\hline
\end{tabular}

PI3K/AKT/mTOR inhibitors and heat shock protein 90 (HSP90) inhibitors. Understanding the signaling pathways of the HER receptors may contribute positively to developing specific inhibitors of several molecular pathways. The PI3K/ $\mathrm{AKT} / \mathrm{mTOR}$ pathway is the main pathway being investigated for anti-HER2 therapies. It is known that PIK3CA mutations occur in about one-third of patients with HER2-positive breast cancers. This may be related to worse prognosis [40]. 
Combinations of various inhibitors of this pathway with standard treatments have been tested or are still being tested in several clinical trials. Two clinical trials (BOLERO-1 and BOLERO-3) have not confirmed the effectiveness of the combination of mTOR inhibitor everolimus with standard anti-HER2 therapies [41, 42]. However, subsequent analysis of these two trials suggests that some patients (for example, with PIK3CA mutations) may benefit from everolimus [43].

Alpelisib is the next representative of this group of agents. It is an $\alpha$-specific PI3K inhibitor. Combining alpelisib and T-DM1 resulted in 10.6 months of median PFS in phase I trial, and will be continued in phase II [3]. Another phase I trial on alpelisib in combination with trastuzumab and HER3 inhibitor LJM716 in patients with HER2-positive advanced $\mathrm{BC}$ is ongoing (ClinicalTrials.gov identifier: NCT02167854) [44]. Other PI3K inhibitors being currently evaluated are copanlisib (ClinicalTrials.gov identifier: NCT02705859), taselisib (ClinicalTrials.gov identifier: NCT02390427) or buparlisib [3, 45, 46]. None of PI3K inhibitors is currently registered.

The regulation of HER2 receptors expression is another approach to anti-HER 2 therapy. There were presented some encouraging results when ganetespib (heat shock protein 90 (HSP90) inhibitor) was combined with trastuzumab and paclitaxel administered once a week in MBC cases. In two of nine patients, partial tumour response was obtained (overall response rate: $22 \%$ ), five patients achieved disease stability (rate: 56\%), which lasted from $11-29$ weeks, clinical benefit rate $-44 \%$. The combination of these three drugs warrants further study [47].

Bispecific antibodies (BsAbs), defined as agents capable of binding to two different types of antigen simultaneously, for instance, an antigen of cytotoxic cell and an antigen expressed by a cancer cell. This may be the next mechanism of action of anticancer drugs. The main representative of BsAbs is ertumaxomab. This is a trifunctional BsAb that targets HER2, CD3 antigen specific to T-cells and the Fc囚-receptors I, IIa, and III. The first trial of ertumaxomab showed an antitumour response in $33 \%$ of patients. Further investigation of ertumaxomab is required [19]. Other representatives of BsAbs under clinical development are MCLA-128, ZW25, GBR1302, PRS-343 [10].

CDK4/6 inhibitors. The addition of CDK4/6 inhibitors to the standard treatment of HER2-positive BC may have a positive influence in overcoming acquired resistance to anti-HER2 therapies [48]. Two main clinical studies investigating the role of these agents in HER2-positive BC are the PATINA study and the MonarcHER study. The PATINA study assesses the usage of palbociclib in combination with trastuzumab, pertuzumab and an aromatase inhibitor [49]. The MonarcHer study is addressed to previously treated MBC patients and explores the influence of the combination of abemaciclib and trastuzumab [50].

HER2-targeting vaccines. HER2-targeting vaccines are being evaluated at present in several clinical trials. One is testing the HER2 vaccine NeuVaxTM, which consists of Nelipepimut-S or E75 peptide and granulocyte macrophagecolony stimulating factor. The study is evaluating the use of this vaccine in combination with trastuzumab in HER2-positive BC patients (ClinicalTrials.gov identifier: NCT02297698) [51]. Another vaccine under investigation in a phase I trial is ETBX-021. The role of this vaccine is being tested in low-expressing HER2 BC patients [10].

\section{CONCLUSIONS}

The development of possible methods of treatment in oncology results in prolongation of the time of survival among patients suffering from malignancies. Breast cancer patients also experience increasingly better outcomes. Due to the discovery of HER2-targeting therapies, a significant improvement is observed in PFS and OS among HER2positive BC patients. Access to drugs, such as trastuzumab, pertuzumab, lapatinib and T-DM1, enables patients to live a relatively normal life, even in those with advanced disease. Moreover, final analysis of the some clinical trials show that breast cancer is becoming the chronic desease. Novel drugs are being evaluated in many clinical trials and many of them are already showing promising results. It is hoped that in the future it may be possible to cure even metastatic cases of breast cancer.

\section{REFERENCES}

1.https://www.iarc.fr/featured-n... assesed at January 6th, 2020.

2. Krishnamurti U, Silverman JF. HER2 in Breast Cancer: A Review and Update. Adv Anat Pathol. 2014; 21(2): 100-107. doi: 10.1097/ PAP.0000000000000015

3. Escriva-de-Romaní S, Arumí M, Bellet M, Saura C. HER2-positive breast cancer: Current and new therapeutic strategies. Breast. 2018; 39: 80-88. doi: $10.1016 /$ j.breast.2018.03.006

4. Wolff AC, Hammond ME, Hicks DG, Dowsett M, McShane LM, Allison $\mathrm{KH}$, et al. Recommendations for human epidermal growth factor receptor 2 testing in breast cancer: American Society of Clinical Oncology/College of American Pathologists clinical practice guideline update. J Clin Oncol. 2013; 31(31): 3997-4013. doi: 10.1200/ JCO.2013.50.9984

5. Veeraraghavan J, De Angelis C, Reis-Filho JS, Pascual T, Prat A, Rimawi MF, et al. De-escalation of treatment in HER2-positive breast cancer: determinants of response and mechanisms of resistance. Breast. 2017; 34(1): 19-26. doi: 10.1016/j.breast.2017.06.022

6. Yao M, Fu P. Advances in anti-HER2 therapy in metastatic breast cancer. Chin Clin Oncol. 2018; 7(3): 27. doi: 10.21037/cco.2018.05.04

7. Albanell J, Baselga J. Trastuzumab, a humanized anti-HER2 monoclonal antibody, for the treatment of breast cancer. Drugs Today (Barc). 1999; 35(12): 931-946.

8. Slamon DJ, Leyland-Jones B, Shak S, Fuchs H, Paton V, Bajamonde A, et al. Use of chemotherapy plus a monoclonal antibody against HER2 for metastatic breast cancer that overexpresses HER2. N Engl J Med. 2001; 344(11): 783-792.

9.von Minckwitz G, du Bois A, Schmidt M, Maass N, Cufer T, de Jongh FE, et al. Trastuzumab beyond progression in human epidermal growth factor receptor 2-positive advanced breast cancer: a german breast group 26/breast international group 03-05 study. J Clin Oncol. 2009; 27(12): 1999-2006. doi: 10.1200/JCO.2008.19.6618

10. Pernas S, Tolaney SM. HER2-positive breast cancer: new therapeutic frontiers and overcoming resistance. Ther Adv Med Oncol. 2019; 11: 1-16. doi: 10.1177/1758835919833519

11. Cortés J, Fumoleau P, Bianchi GV, Petrella TM, Gelmon K, Pivot X, et al. Pertuzumab monotherapy after trastuzumab-based treatment and subsequent reintroduction of trastuzumab: activity and tolerability in patients with advanced human epidermal growth factor receptor 2-positive breast cancer. J Clin Oncol. 2012; 30(14): 1594-1600. doi: 10.1200/JCO.2011.37.4207

12. Baselga J, Cortes J, Kim SB, Im SA, Hegg R, Im YH, et al. Pertuzumab plus trastuzumab plus docetaxel for metastatic breast cancer. N Engl J Med. 2012; 366(2): 109-119. doi: 10.1056/NEJMoa1113216 
13. Swain SM, Baselga J, Kim SB, Ro J, Semiglazov V, Campone M et al. Pertuzumab, trastuzumab, and docetaxel in HER2-positive metastatic breast cancer. N Engl J Med. 2015; 372(8): 724-734. doi: 10.1056/ NEJMoa1413513

14. HelwickC.CLEOPATRAEnd-of-StudyAnalysisinMetastaticBreastCancer: $37 \%$ Alive at 8 Years. www.ascopost.com/issues/july-10-2019/cleopatraend-of-study-analysis-in-metastatic-breast-cancer/?fbclid=IwAR311s voKc3dfzuVM-qWTvEpgf24TIcrjUc2fiZbS1Fe_EYc3oT8tpqs4M

15. Bang YJ, Giaccone G, Im SA, Oh DY, Bauer TM, Nordstrom JL, et al. First-in-human phase 1 study of margetuximab (MGAH22), an Fcmodified chimeric monoclonal antibody, in patients with HER2-positive advanced solid tumors. Ann Oncol. 2017; 28(4): 855-861. doi: 10.1093/ annonc/mdx002

16. ClinicalTrials.gov. Margetuximab Plus Chemotherapy vs Trastuzumab Plus Chemotherapy in the Treatment of HER2+ Metastatic Breast Cancer (SOPHIA). www.clinicaltrials.gov/ct2/show/NCT02492711

17. Segovia-Mendoza M, Gonzalez-Gonzalez ME, Barrera D, Diaz L, Garcia-Becerra R. Efficacy and mechanism of action of the tyrosine kinase inhibitors gefitinib, lapatinib and neratinib in the treatment of HER2-positive breast cancer: preclinical and clinical evidence. Am J Cancer Res. 2015; 5(9): 2531-2561.

18. Geyer CE, Forster J, Lindquist D, Chan S, Romieu CG, Pienkowski T, et al. Lapatinib plus capecitabine for HER2-positive advanced breast cancer. N Engl J Med. 2006; 355(26): 2733-2743.

19. Gligorov J, Richard S, Todorovic V. New anti-HER2 agents: from secondgeneration tyrosine kinases inhibitors to bifunctional antibodies. Curr Opin Oncol. 2017;29(6): 405-410. doi: 10.1097/CCO.0000000000000412

20. Burstein HJ, Sun Y, Dirix LY, Jiang Z, Paridaens R, Tan AR, et al. Neratinib, an irreversible ErbB receptor tyrosine kinase inhibitor, in patients with advanced ErbB2-positive breast cancer. J Clin Oncol. 2010; 28(8): 1301-1307. doi: 10.1200/JCO.2009.25.8707

21. Saura C, Garcia-Saenz JA, Xu B, Harb W, Moroose R, Pluard T, et al. Safety and efficacy of neratinib in combination with capecitabine in patients with metastatic human epidermal growth factor receptor 2-positive breast cancer. J Clin Oncol. 2014; 32(32): 3626-3633. doi: 10.1200/JCO.2014.56.3809

22. Awada A, Colomer R, Inoue K, Bondarenko I, Badwe RA, Demetriou G, et al. Neratinib Plus Paclitaxel vs Trastuzumab Plus Paclitaxel in Previously Untreated Metastatic ERBB2-Positive Breast Cancer: The NEfERT-T Randomized Clinical Trial. JAMA Oncol. 2016; 2(12): 1557-1564. doi: 10.1001/jamaoncol.2016.0237

23. Chan A, Delaloge S, Holmes FA, Moy B, Iwata H, Harvey VJ, et al. Neratinib after trastuzumab-based adjuvant therapy in patients with HER2-positive breast cancer (ExteNET): a multicentre, randomised, double-blind, placebo-controlled, phase 3 trial. Lancet Oncol. 2016; 17(3): 367-377. doi: 10.1016/S1470-2045(15)00551-3

24. ClinicalTrials.gov. A Study of Neratinib Plus Capecitabine Versus Lapatinib Plus Capecitabine in Patients With HER2+ Metastatic Breast Cancer Who Have Received Two or More Prior HER2 Directed Regimens in the Metastatic Setting (NALA). www.clinicaltrials.gov/ ct $2 /$ show/NCT01808573

25. Cortés J, Dieras V, Ro J, Berriere J, Bachelot T, Hurvitz S, et al. Afatinib alone or afatinib plus vinorelbine versus investigator's choice of treatment for HER2-positive breast cancer with progressive brain metastases after trastuzumab, lapatinib, or both (LUX-Breast 3): a randomised, open-label, multicentre, phase 2 trial. Lancet Oncol. 2015; 16(16): 1700-1710. doi: 10.1016/S1470-2045(15)00373-3

26. ClinicalTrials.gov. A Study of Tucatinib vs. Placebo in Combination With Capecitabine \& Trastuzumab in Patients With Advanced HER2+ Breast Cancer (HER2CLIMB). https://clinicaltrials.gov/ct2/show/NCT02614794

27. Verma S, Miles D, Gianni L, Krop IE, Welslau M, Baselga J, et al. Trastuzumab emtansine for HER2-positive advanced breast cancer. N Engl J Med. 2012; 367(19): 1783-1791. doi: 10.1056/NEJMoa1209124

28. Diéras V, Miles D, Verma S, Pegram M, Welslau M, Baselga J, et al. Trastuzumab emtansine versus capecitabine plus lapatinib in patients with previously treated HER2-positive advanced breast cancer (EMILIA): a descriptive analysis of final overall survival results from a randomised, open-label, phase 3 trial. Lancet Oncol. 2017; 18(6): 732-742. doi: 10.1016/S1470-2045(17)30312-1

29. Krop IE, Kim SB, Martin AG, LoRusso PM, Ferrero JM, BadowinacCrnjevic T, et al. Trastuzumab emtansine versus treatment of physician's choice in patients with previously treated HER2-positive metastatic breast cancer (TH3RESA): final overall survival results from a randomised open-label phase 3 trial. Lancet Oncol. 2017; 18(6): 743-754. doi: 10.1016/S1470-2045(17)30313-3

30. Perez EA, Barrios C, Eiermann W, Toi M, Im YH, Conte P, et al. Trastuzumab emtansine with or without pertuzumab versus trastuzumab plus taxane for human epidermal growth factor receptor 2-positive, advanced breast cancer: primary results from the phase III MARIANNE study. J Clin Oncol. 2017; 35(2): 141-148.

31. Doi T, Shitara K, Naito Y, Shimomura A, Fujiwara Y, Yonemori K, et al. Safety, pharmacokinetics, and antitumour activity of trastuzumab deruxtecan (DS-8201), a HER2-targeting antibody-drug conjugate, in patients with advanced breast and gastric or gastrooesophageal tumours: a phase 1 dose-escalation study. Lancet Oncol. 2017; 18(11): 1512-1522. doi: 10.1016/S1470-2045(17)30604-6

32. ClinicalTrials.gov. DS-8201a in Pre-treated HER2 Breast Cancer That Cannot be Surgically Removed or Has Spread [DESTINY-Breast02]. www.clinicaltrials.gov/ct2/show/NCT03523585.

33. ClinicalTrials.gov. DS-8201a Versus T-DM1 for Human Epidermal Growth Factor Receptor 2 (HER2)-Positive, Unresectable and/or Metastatic Breast Cancer Previously Treated With Trastuzumab and Taxane [DESTINYBreast03]. www.clinicaltrials.gov/ct2/show/NCT03529110.

34. ClinicalTrials.gov. SYD985 vs. Physician's Choice in Participants With HER2-positive Locally Advanced or Metastatic Breast Cancer (TULIP). www.clinicaltrials.gov/ct2/show/NCT03262935.

35. ClinicalTrials.gov. Study of RC48-ADC in Patients With HER2-Positive Advanced Malignant Solid Tumors. www.clinicaltrials.gov/ct2/show/ NCT02881138

36. ClinicalTrials.gov. PF-06804103 Dose Escalation in HER2 Positive Solid Tumors. www.clinicaltrials.gov/ct2/show/NCT03284723.

37. ClinicalTrials.gov. A Dose-escalation Study of ARX788, IV Administered in Subjects With Advanced Cancers With HER2 Expression. www. clinicaltrials.gov/ct2/show/NCT02512237.

38. ClinicalTrials.gov. A Phase 1/2 Study of MEDI4276 in Adults Subjects With Select HER2-expressing Advanced Solid Tumors. (MEDI4276). www.clinicaltrials.gov/ct2/show/NCT02576548.

39. ClinicalTrials.gov. Study of Antibody Drug Conjugate in Patients With Advanced Breast Cancer Expressing HER2. www.clinicaltrials.gov/ct2/ show/NCT02952729.

40. The Cancer Genome Atlas Network. Comprehensive molecular portraits of human breast tumours. Nature. 2012; 490(7418): 61-70. doi: 10.1038/ nature11412

41. Hurvitz SA, Andre F, Jiang Z, Shao Z, Mano MS, Neciosup SP, et al. Combination of everolimus with trastuzumab plus paclitaxel as firstline treatment for patients with HER2-positive advanced breast cancer (BOLERO-1): a phase 3, randomised, double-blind, multicentre trial. Lancet Oncol. 2015; 16(7): 816-829. doi: 10.1016/S1470-2045(15)00051-0 42. Andre'F, O’Regan R, Ozguroglu M, Toi M, Xu B, Jerusalem G, et al. Everolimus for women with trastuzumab-resistant, HER2-positive, advanced breast cancer (BOLERO-3): a randomised, double-blind, placebo-controlled phase 3 trial. Lancet Oncol. 2014; 15(6): 580-591. doi: 10.1016/S1470-2045(14)70138-X

43. Andre' F, Hurvitz S, Fasolo A, Tseng LM, Jerusalem G, Wilks S, et al. Molecular alterations and everolimus efficacy in human epidermal growth factor receptor 2-overexpressing metastatic breast cancers: combined exploratory biomarker analysis from BOLERO-1 and BOLERO-3. J Clin Oncol. 2016; 34(18): 2115-2124. doi: 10.1200/JCO.2015.63.9161

44. ClinicalTrials.gov. Open-Label Study Evaluating the Safety and Tolerability of LJM716, BYL719 and Trastuzumab in Patients With Metastatic HER2+ Breast Cancer. www.clinicaltrials.gov/ct2/show/ NCT02167854

45. ClinicalTrials.gov. Phase Ib/II Trial of coPANlisib in Combination With Trastuzumab in HER2-positive Breast Cancer. (Panther Study) (Panther). www.clinicaltrials.gov/ct2/show/NCT02705859

46. ClinicalTrials.gov. Phase Ib Dose-escalation Trial of Taselisib (GDC0032) in Combination With Anti-HER2 Therapies in Participants With Advanced HER2+ Breast Cancer. www.clinicaltrials.gov/ct2/ show/NCT02390427

47. Jhaveri K, Wang R, Teplinsky E, Chandarlapaty S, Solit D, Cadoo K, et al. A phase I trial of ganetespib in combination with paclitaxel and trastuzumab in patients with human epidermal growth factor receptor-2 (HER2)-positive metastatic breast cancer. Breast Cancer Res. 2017; 19(1): 89. doi: 10.1186/s13058-017-0879-5

48. Goel S, Wang Q, Watt AC, Tolaney SM, Dillon DA, Li W, et al. Overcoming therapeutic resistance in HER2-positive breast cancers with CDK4/6 inhibitors. Cancer Cell. 2016; 29(3): 255-269. doi: 10.1016/j. ccell.2016.02.006

49. ClinicalTrials.gov, Randomized, Open Label, Clinical Study of the Targeted Therapy, Palbociclib, to Treat Metastatic Breast Cancer (PATINA), https://clinicaltrials.gov/ct2/show/NCT02947685

50. ClinicalTrials.gov, A Study of Abemaciclib (LY2835219) in Women With HR+, HER2+ Locally Advanced or Metastatic Breast Cancer (monarcHER), https://clinicaltrials.gov/ct2/show/NCT02675231

51. ClinicalTrials.gov, Phase II Trial of Combination Immunotherapy With NeuVax and Trastuzumab in High-risk HER2+ Breast Cancer Patients (HER3+), https://clinicaltrials.gov/ct2/show/NCT02297698 